\title{
Enactment and retrieval
}

\author{
Daniel J. Peterson and Neil W. Mulligan \\ University of North Carolina, Chapel Hill, North Carolina
}

\begin{abstract}
The enactment effect is one of a number of effects (e.g., bizarreness, generation, perceptual interference) that have been treated in common theoretical frameworks, most of them focusing on encoding processes. Recent results from McDaniel, Dornburg, and Guynn (2005) call into question whether bizarreness and, by association, related phenomena such as enactment are better conceptualized as arising due to retrieval processes. Four experiments investigated the degree to which retrieval processes are responsible for enhanced memory for enacted phrases. Participants were presented with two pure study lists and later recalled the lists separately (inducing pure retrieval sets) or recalled the lists together in a single test (inducing a combined or mixed retrieval set). Across all four experiments, the combined recall condition consistently failed to enhance the size of the enactment effect. The results provide no support for the retrieval account but are generally consistent with encoding accounts.
\end{abstract}

Although much of the research on human memory has focused on verbal materials, a significant portion of what people actually remember relates to what they have done. Research on enactment, or action memory, is concerned with understanding how people recall both simple and complex action sequences and how these results relate to those of verbal materials, a more thoroughly researched area. In a standard paradigm for studying action memory, participants perform simple tasks (such as tapping a desk or breaking a pencil), watch an experimenter perform the same task, or read/hear a description of the task. Tasks carried out by the participant (subject-performed tasks, or SPTs) usually result in better memory than do either experimenter-performed tasks (EPTs) or verbal tasks (VTs), a phenomenon dubbed the enactment or SPT effect (Engelkamp, 1998). The enactment effect has been found on a variety of tests, including recognition (e.g., Engelkamp, Zimmer, Mohr, \& Sellen, 1994), free recall (e.g., Engelkamp \& Dehn, 2000), and cued recall (e.g., Kormi-Nouri, 1995).

Several early studies indicated that enactment provides an efficient, nonstrategic encoding process that differed in some ways from the encoding of verbal information (Cohen, 1981, 1989; Engelkamp \& Zimmer, 1985). For example, SPTs are less likely to produce serial position effects in recall (Cohen, 1981) or age effects than are VTs (Bäckman \& Nilsson, 1984). Other research by Engelkamp and colleagues indicates that the motor component of SPTs is critical for the memorial benefits of enactment (e.g., Engelkamp, 1998). One particularly compelling demonstration has come from Engelkamp et al. (1994), in which enactment was manipulated not only during encoding, but at retrieval as well. When participants reenacted a phrase at test, recognition improved (see also Mulligan \& Hornstein, 2003). This reenactment effect was interpreted in terms of the encoding specificity principle (e.g., Tulving \& Thomson, 1973) and was taken as evidence that motor information was a critical part of the original memory trace for SPTs. Further support of the motor component theory has come from studies demonstrating an SPT advantage for blind participants and for normally sighted but blindfolded participants (KormiNouri, 2000; Mulligan \& Hornstein, 2003), indicating that visual imagery or visual feedback does not drive the SPT effect. Similarly, several researchers have required participants to interact with imaginary rather than actual objects (e.g., Engelkamp \& Zimmer, 1984; Kormi-Nouri, 2000). Enactment still improves memory, refuting the possibility that it is simply the tactile feedback of the object in one's hand that improves memory.

Despite some differences between memory for actions and memory for verbal materials, there are also a number of important similarities. Consequently, the SPT effect has been analyzed in terms of two related memory frameworks: the item-specific-relational account and the item order account, both of which apply to memory for verbal materials, as well as to memory for actions.

When learning a list of items, participants do not merely encode each item individually; associations are also formed among the items. Hunt and McDaniel (1993) outlined the item-specific-relational framework to differentiate these two types of processing. Item-specific processing is defined as the processing of features unique to a stimulus, increasing the item's distinctiveness in memory. Relational processing, conversely, is defined as the analyses of shared features, such as the connections made between cues and targets, associations among target items, and associations between individual items and the list as

N.W. Mulligan, nmulligan@unc.edu 
a whole. Most researchers agree that enactment enhances item-specific processing, as demonstrated by consistent SPT effects in recognition memory (e.g., Engelkamp \& Dehn, 2000; Engelkamp et al., 1994; Steffens, 2007), a test thought to rely heavily on item-specific processing. Conversely, enactment generally appears to leave unaffected or to hinder relational processing (e.g., Bäckman, Nilsson, \& Chalom, 1986; Steffens, 2007; Steffens, Buchner, \& Wender, 2003), which helps account for some of the null and negative effects of enactment mentioned below. In a related framework, McDaniel and Bugg (2008) outlined the similarities between the enactment effect and several other effects, arguing that all are accommodated by a common theory. Specifically, the authors delineated how the effects demonstrated with generation (improved memory for generated material), perceptual interference (improved memory for items presented only very briefly), bizarreness (improved memory for bizarre or uncommon sentences), enactment, and word frequency can all be understood in terms of the item order account (Nairne, Riegler, \& Serra, 1991).

For all five of these variables, the effect on recall depends on list composition. For example, in the case of generation, when participants are required to generate an item themselves, they typically show increased recall for the item, as compared with having simply read it (e.g., Mulligan \& Lozito, 2004). However, this effect depends on whether the variable was manipulated in a mixed-list or a pure-list design. In the former case, the study list consists of generate items randomly interspersed with read items, whereas in the latter case, the study list is entirely generate items or entirely read items. Typically, the generation effect in recall is robust in mixed-list designs but smaller (or nonexistent) in pure-list designs.

The standard account of the pure- versus mixed-list effect is articulated in terms of item-specific and relational (or order) information (Hunt \& McDaniel, 1993; McDaniel \& Bugg, 2008; Steffens, 2007; see also Roediger, 2008). This account argues that item-specific, as well as relational, processing aids in free recall. Relational information provides the structure to guide retrieval, whereas item-specific information provides discriminative information among potential responses. The item order account focuses on order information as a specific type of relational information that is particularly important in guiding recall (e.g., Nairne et al., 1991).

In regard to the mixed- versus pure-list distinction, the nature of the list composition differentially affects itemspecific and relational encoding. Each variable (e.g., bizarreness, enactment, generation, perceptual interference, etc.) contrasts an unusual and a common condition. Unusual stimuli, such as bizarre sentences or items to be generated, attract more attention for interpretation, resulting in greater item processing. This enhancement, however, comes at the cost of the encoding of relational information, since encoding resources are drawn to the encoding of item characteristics and away from the encoding of relations among target items. More usual stimuli, such as common sentences or read items, attract less process- ing of item characteristics, allowing for greater processing of interitem associations. During mixed-list presentations of both generate and read items, for example, the disruption of relational processing caused by the presentation of generate items uniformly hinders recall of both read and generate items. However, the generate items uniquely benefit from the enhancement in item processing, leading to the recall benefits seen with generated material. In pure lists of generate items, the reduction of relational processing counteracts any item-specific improvements, resulting in a decreased or eliminated recall advantage with respect to the read items.

Because recognition is primarily sensitive to itemspecific, and not relational, processing, this view predicts a robust effect of the variable (generation, bizarreness, enactment, etc.) in recognition for both mixed and pure lists (in contrast to results with free recall). This is the typical finding for all the manipulations. In contrast, more direct measures of relational or order information usually indicate worse performance for the unusual conditions, relative to the common conditions (e.g., Engelkamp \& Dehn, 2000; McDaniel \& Bugg, 2008; Mulligan, 1999; Mulligan \& Lozito, 2004; Nairne et al., 1991; Steffens, 2007), which supports the theory and underscores the commonality across the five variables.

As is true for all the variables in this set (bizarreness, generation, perceptual interference, enactment, etc.), the standard accounts of the enactment effect focus on encoding processes. That is, the differential item processing believed to underlie these effects takes place during initial encoding, and, it is assumed, later recall simply reveals this differential encoding. In contrast, these effects have also been framed in terms of a retrieval explanation that emphasizes retrospective distinctiveness (Hunt \& Worthen, 2006; Schmidt, 2007). More specifically, this view proposes that encoding conditions interact with the retrieval context to produce observable effects. Under this view, the unusual encoding condition (e.g., generation, bizarre imagery, enactment) is thought to make items more distinctive in memory. However, the manifestation of distinctiveness is argued to be dependent on the set of items that are recruited as part of the retrieval set (McDaniel, Dornburg, \& Guynn, 2005). Retrieval set refers to the set of items that share a common spatiotemporal context. In an experiment, this context is usually specified (at least implicitly) by the task instructions (e.g., "recall the words from the list you just read"). More generally, it is assumed that all episodic retrieval involves a retrieval set constrained by the retrieval request (Hunt \& McDaniel, 1993).

The retrieval account proposes that if all of the items in the retrieval set share the same features, there is no relative distinctiveness. Applied to design effects, the pure-list condition produces a retrieval set in which all of the items are of the same type and, thus, none is relatively distinct. A lack of relative distinctiveness produces the diminished or null effects in recall. In the mixed-list condition, the retrieval set encompasses an assortment of common and unusual items, conferring relative distinctiveness onto 
the unusual condition, which in turn produces the typical effect in recall. A distinctiveness-based retrieval account has been proposed for several variables that are subject to design effects, such as generation and bizarreness (Begg, Snider, Foley, \& Goddard, 1989; McDaniel, DeLosh, \& Merritt, 2000; McDaniel et al., 2005; Schmidt, 2007).

McDaniel et al. (2005) recently analyzed the bizarreness effect from the perspective of retrieval-based distinctiveness, offering an explanation of design effects that differs markedly from the usual encoding accounts. McDaniel et al. (2005) pointed out that design effects are ambiguous with respect to the distinction between encoding and retrieval accounts. In the typical paradigm, a participant is presented with a mixed or pure study list, followed by a recall test. In a mixed-list presentation, participants read both common and bizarre sentences; that is, the list is mixed with respect to encoding. However, because the retrieval set consists of all of the study sentences, both common and bizarre, the retrieval set is likewise mixed. In this mixed-list presentation, the unusual material is relatively distinct, explaining why an effect is found. In a pure-list presentation, there is also a pure retrieval set, meaning that no items are relatively distinct, which is why no effect is demonstrated. The typical design, however, cannot differentiate between an encoding and a retrieval account, because the mixed versus pure encoding is confounded with the mixed versus pure retrieval set.

To disentangle this confound, McDaniel et al. (2005) presented participants with two pure lists of bizarre or common sentences, separated by a distractor task. At test, the participants were placed in one of two retrieval conditions. In the combined recall condition, the participants were instructed to recall any of the sentences presented in either list during a 5-min free recall session. In the separate recall condition, the participants were given $2.5 \mathrm{~min}$ to recall all of the items presented in one list, then $2.5 \mathrm{~min}$ to recall any of the items presented in the other list. From the perspective of encoding accounts, no difference between the two test conditions would be expected because the encoding conditions (i.e., the purelist presentation) were held fixed. However, according to the retrieval account, the testing conditions should affect the retrieval set. Specifically, the separate recall condition should yield relatively homogeneous retrieval sets, producing little relative distinctiveness and, thus, little effect of bizarreness. In contrast, the combined test should produce a mixed retrieval set, consisting of items from both lists (and both sentence types), conferring relative distinctiveness upon the bizarre items and inducing a bizarreness effect. The results were consistent with McDaniel et al.'s (2005) retrieval account: A bizarreness effect was found in the combined condition, but not in the separate condition.

McDaniel et al.'s (2005) results are important for several reasons. First, design effects have generally been interpreted in terms of encoding accounts (McDaniel \& Bugg, 2008; Roediger, 2008). McDaniel et al.'s (2005) results support a retrieval-based alternative. Second, as was noted earlier, design effects are theoretically impor- tant for a number of experimental variables. McDaniel et al.'s (2005) results imply that our understanding of design effects may be incomplete. Third, these results imply that related variables, such as enactment, generation, and perceptual interference, may be amenable to a similar retrieval-based account. Because these variables share a number of empirical regularities (e.g., design effects, their effects on order, and relational information; McDaniel \& Bugg, 2008; Mulligan \& Lozito, 2004), it may be the case that the design effects found with enactment, like bizarreness, are amenable to this new retrievalbased explanation. The present study tested the generality of McDaniel et al.'s (2005) findings with respect to enactment.

Mulligan and Peterson (2008), employing the design of McDaniel et al. (2005), found that the retrieval account could not adequately explain the effects of perceptual interference or generation. That is, the effects of these variables were not moderated by the manipulation of combined versus separate recall tests. It is critical to extend the analysis to enactment, for several reasons. (1) Examining the contributions of retrieval processes in enactment provides information on an additional variable in the set producing design effects. (2) Action phrases are more comparable to the bizarre sentences used in McDaniel et al. (2005). One possibility raised by Mulligan and Peterson to account for the discrepant results between the two studies was that, in their study, the stimuli (words) were much more atomistic than the sentence stimuli used in McDaniel et al. (2005). If this distinction is important, the present study should serve as a much more appropriate comparison. (3) Finally, and perhaps most importantly, enactment is unique relative to the other variables producing design effects, due to the relative automaticity demonstrated at encoding. Typically, enactment does not yield the same primacy, levels of processing, rehearsal, or directed-forgetting effects (Earles \& Kersten, 2002; Engelkamp, 1998) as those seen with verbal materials. These characteristics imply that action memory is less subject to controlled encoding processes, raising the possibility that the enactment advantage arises due to factors operative at retrieval.

\section{EXPERIMENT 1}

Experiment 1 used the procedures of McDaniel et al. (2005). Participants were presented with two lists of action phrases, one of which was presented in the SPT condition, the other in the VT condition. The participants were later instructed to recall those phrases either in two separate recall periods or in one combined recall test. According to the retrieval hypothesis, the separate recall condition should produce retrieval sets that are homogeneous with respect to the SPT or VT condition and, therefore, should produce little or no effect of SPT on recall. In contrast, the combined recall condition should produce a mixed retrieval set, producing an SPT effect. Thus, the retrieval account predicts an interaction between the encoding and retrieval conditions. Conversely, encoding accounts sug- 
gest no difference between the recall conditions, because no factors vary at encoding.

Because the retrieval account predicts an interaction between the SPT effect and test condition, it is important to maximize the potential for an SPT effect. To do so, VTs served as the controls. Employing VTs as the control for SPTs typically yields larger enactment effects, relative to other controls such as EPTs (e.g., Engelkamp \& Zimmer, 1985). In addition, all SPTs were enacted with real objects. Experiments on action memory have sometimes used real objects and other times imaginary objects. Both produce the SPT effect, but the effect may be larger when real objects are used (e.g., Steffens et al., 2003).

\section{Method}

Participants. Thirty-two undergraduates at the University of North Carolina at Chapel Hill served as participants in exchange for course credit.

Design. The experiment used a 2 (encoding condition: SPT vs. VT) $\times 2$ (recall condition: combined vs. separate) design in which the encoding condition was manipulated within participants and the recall condition was manipulated between participants.

Materials. Thirty-two simple action phrases were taken from Mulligan and Hornstein (2003; see the Appendix), following the guidelines set forth by Steffens et al. (2003). ${ }^{1}$ No object or action was repeated across items. Each of the phrases was randomly assigned to one of two lists, resulting in two lists of 16 items each. Each list was presented in the SPT or VT condition, counterbalanced across participants. Two additional items were placed at the beginning and end of each study list to serve as practice items and buffers, resulting in two study lists of 20 items each.

Procedure. All the participants were tested individually in a lab room with a small conference table. The experimenter and the participant sat facing each other so that each had a clear view of the other. The study phase began with the first list of phrases. The participants were informed that they would hear a series of phrases describing simple actions and that their task was either to perform the action or simply to listen to the action. In addition, the participants were told to try to remember the phrases for a later, unspecified memory test. Each list consisted entirely of SPT or VT phrases. The order of the lists was counterbalanced across participants. In the SPT condition, each trial proceeded as follows. First, the relevant object was brought out and placed on the table in view of the participants. Next, the action phrase was presented over the computer speaker. Then, the participants acted out the phrase, interacting with the object (e.g., actually breaking a pencil). After $8 \mathrm{sec}$, the item was removed, and the next trial began. The VT trials followed the same procedure, except that the participants only listened to the phrases, rather than acting them out. The participants were still presented with the object; they simply did not interact with it. After the presentation of each list, the participants carried out a distractor task for $5 \mathrm{~min}$. The participants were presented with a sheet of math problems to solve in their heads without making any notes or intermediate calculations on the paper.

At test, the participants were randomly placed into one of two recall conditions. In the combined condition, the participants were instructed to recall as many of the phrases as they could from both lists, both the VT and SPT phrases. This test lasted $5 \mathrm{~min}$, and the participants were encouraged to use the entire time allotted. In the separate recall condition, the participants were instructed to think back and recall all the items remembered from one list (the order of which was counterbalanced across participants) for $2.5 \mathrm{~min}$. Following this, they were given a second recall test in which they were given an additional 2.5 min to think back and recall all the items remembered from the other list. In the instructions of each test, the participants were reminded of the nature of the items for the to-be- recalled list (e.g., the participants were asked to recall List 1 and were reminded that this was the list in which they had acted out the phrases).

\section{Results and Discussion}

Recall performance was scored in two ways. The stringent recall score required that both the noun and verb of a phrase be recalled together to count as correct (e.g., break and pencil for break the pencil). For the lenient score, an item was considered correctly recalled if either the noun or the verb was recalled. Both scores yielded the same pattern of results in this and later experiments; only the lenient recall scores are reported. ${ }^{2}$ The proportions recalled (Table 1 ) were submitted to a $2 \times 2$ ANOVA, using encoding condition (SPT or VT) and recall test (combined or separate) as factors ( $\alpha=.05$ for this and subsequent analyses). The analysis revealed a main effect for recall condition $\left[F(1,30)=6.35, M S_{\mathrm{e}}=23.77\right]$, indicating higher recall in the combined than in the separate condition. The effect of encoding condition and the interaction were not significant $(p \mathrm{~s}>.25)$.

Before considering the more critical results, we will discuss the unexpected main effect of recall condition. It is possible that the combined condition produced higher recall because it led to a functionally shorter retention interval for the list that was to be recalled second in the separate condition. That is, if the participants in the combined condition completed the bulk of their recall in less than $2.5 \mathrm{~min}$ (which seems likely on the basis of typical recall dynamics; e.g., Mulligan, 2006), they largely finished recalling both lists before the separate condition would begin on their second test (although the analysis in note 2 argues against this). However, earlier research using this paradigm (McDaniel et al., 2005; Mulligan \& Peterson, 2008) did not show significant main effects of recall condition. Likewise, no main effect of recall condition was found in the subsequent experiments of the present project. Consequently, this effect will be treated as a nonreplicable result and will not be discussed further.

According to McDaniel et al.'s (2005) analysis, the separate recall condition should be no different from traditional pure-list designs. Because pure-list designs often

Table 1

Mean Proportions (With Standard Deviations) of Items Recalled As a Function of Encoding and Recall Conditions

\begin{tabular}{|c|c|c|c|c|}
\hline \multirow{3}{*}{$\begin{array}{c}\text { Recall } \\
\text { Condition }\end{array}$} & \multicolumn{4}{|c|}{ Encoding Condition } \\
\hline & \multicolumn{2}{|c|}{ SPT } & \multicolumn{2}{|c|}{ VT } \\
\hline & $M$ & $S D$ & $M$ & $S D$ \\
\hline \multicolumn{5}{|l|}{ Experiment 1} \\
\hline Separate & .43 & .09 & .38 & .12 \\
\hline Combined & .49 & .17 & .47 & .10 \\
\hline \multicolumn{5}{|l|}{ Experiment 2} \\
\hline Separate & .54 & .13 & .47 & .11 \\
\hline Combined & .58 & .13 & .49 & .12 \\
\hline \multicolumn{5}{|l|}{ Experiment 3} \\
\hline Separate & .45 & .16 & .42 & .13 \\
\hline Combined & .48 & .13 & .45 & .16 \\
\hline
\end{tabular}

Note-SPT, subject-performed task; VT, verbal task. 
do not yield an SPT effect, it is necessary to compare this condition with the combined recall condition in order to induce a combined retrieval set. According to the retrieval account, the SPT effect in the combined condition should be significantly larger than any SPT effect found in the separate condition. The present results provide no evidence for such an interaction.

Although the results appear inconsistent with the retrieval hypothesis, there is room for alternative explanations. One possibility is that perhaps the participants in the separate group were unable to discriminate between the two lists at recall. In other words, if these participants were spontaneously combining both lists at retrieval, then, effectively, there would be no differences in retrieval sets between the two recall groups. However, examining crosslist intrusions (recalling an item from List 1 during the List 2 recall or vice versa) suggests that this was not the case. Not 1 of 16 participants in the separate recall condition made even a single cross-list intrusion, indicating that list discriminability was not a problem.

Alternatively, it is possible that despite the instructions in the combined condition, the participants created two separate retrievals sets, one for the SPT items and a second for the VT items. If this were the case, we would not have satisfied the conditions that would constitute a true combined retrieval set. As such, a failure to demonstrate a significant interaction would not be strong evidence against the retrieval account. To further investigate, we examined list-based clustering in the combined condition. If the participants were spontaneously performing two separate recalls, such a strategy would result in recall clustering by list. To assess this, we used the adjustedratio-of-clustering (ARC) score (Roenker, Thompson, \& Brown, 1971), which has a value of 0 for chance-level clustering, positive values for above-chance clustering, and a value of 1 for perfect clustering. The analysis produced mixed results. For 4 participants, no ARC score could be computed, because these participants spontaneously grouped their responses (i.e., creating two separate columns) on the basis of list. Excluding these 4 participants yielded an average ARC score of .11, indicating little evidence for a separate retrieval strategy for a majority of the participants. It should be noted that restricting analysis to these participants $(n=12)$ versus the separate recall group still produces no hint of an interaction $(F<1)$.

\section{EXPERIMENT 2}

Experiment 1 produced no evidence that the SPT effect emerges at retrieval. The encoding $\times$ recall interaction was nonsignificant, and the ARC scores did not reveal strong evidence for list-based clustering in the combined recall condition that could alternatively explain the null interaction. Nevertheless, Experiment 2 was designed to follow up on the possibility that the two lists were so easily discriminated that the participants in the combined condition were induced to treat their single recall test as two separate recalls. To address this potential problem,
Experiment 2 used two categories of objects, toys and office supplies, which were spread across the two lists. This new categorical structure was designed to provide a salient alternative to list-based recall for the combined recall condition. The participants in this condition could use a category-based retrieval strategy that would render a retrieval set encompassing both lists, providing another test of the retrieval account. Nothing should change during the separate recall, since the list structure still provides an effective retrieval organization when participants are explicitly instructed to recall by list.

\section{Method}

Participants. Thirty-two undergraduates at the University of North Carolina at Chapel Hill participated in exchange for credit in psychology courses.

Design, Materials, and Procedure. Experiment 2 was identical to Experiment 1, except for the following modifications. Sixteen critical phrases were constructed using objects that fell within each of two categories (toys and office supplies; see the Appendix). An additional 4 items from each category were constructed to serve as primacy and recency buffers for the two study lists. A total of 17 items were used from Experiment 1, which fit into one of the two categories. The additional 23 items were newly constructed such that neither an object nor an action was repeated throughout the experiment. The phrases from each category were randomly divided so that half of each category appeared in List 1 and half in List 2 . The study lists were randomly intermixed, with the constraint that items from a single category were repeated no more than twice. The presentation of the SPT and VT lists was identical to that in Experiment 1.

At test, the instructions for the separate condition did not change. The participants in the combined recall condition were informed about the presence of the categories across each list (a detail that had not previously been made explicit) and were encouraged to use these categories to help guide retrieval.

\section{Results and Discussion}

The proportions recalled (Table 1) were submitted to a $2 \times 2$ ANOVA, using encoding condition and recall test as factors. Interestingly, the results revealed a main effect of encoding, such that SPT was greater than VT $[F(1,30)=$ $10.92, M S_{\mathrm{e}}=28.89$; we address this finding in Experiment 4]. There was no main effect of recall test and no significant interaction between encoding condition and type of test $(F \mathrm{~s}<1)$. The separate condition produced minimal cross-list intrusions (a single cross-list intrusion over 16 participants).

Once again, we fail to demonstrate the interaction predicted by the retrieval account. Although the results are consistent with the results of Experiment 1, our category manipulation may not have been as strong as might be desired. By employing categories of items across the two lists, we expected that list-based clustering would be minimized in the combined condition and that category clustering would occur instead. Although there was evidence of category-based clustering [with a mean category-based ARC score of .16, significantly greater than $0 ; t(13)=$ 2.24], there was also evidence of list-based clustering with a mean list-based ARC score of .18 [also significant; $t(13)=2.86]$. The participants were no more likely to cluster on the basis of category than on the basis of list membership $[t(13)=0.144, p>.05]$. 


\section{EXPERIMENT 3}

Both Experiments 1 and 2 produced no evidence that the SPT effect is larger in the combined than in the separate condition. Experiment 2 introduced a category structure across lists to enhance the likelihood of a combined retrieval set in the combined recall condition. Experiment 3 extended this strategy by making category membership even more salient. This was done by presenting samecategory items in blocks within each list.

\section{Method}

Participants. Thirty-two undergraduates at the University of North Carolina at Chapel Hill participated in exchange for credit in psychology courses.

Design, Materials, and Procedure. Experiment 3 was identical to Experiment 2, except for the following modifications. Each study list was rearranged such that the first eight critical items of that list (as well as the two preceding buffer items at the beginning of the list) were all action phrases involving office supplies. The following eight critical items (as well as the two following buffer items at the end of the list) were all action phrases involving toys. Importantly, only the order of these items was changed; no other changes were made from Experiment 2.

\section{Results and Discussion}

The proportions recalled (Table 1) were submitted to a 2 (encoding condition) $\times 2$ (recall condition) ANOVA, which revealed no significant main effects and, again, no interaction $(p s>.4)$. As in the earlier experiments, there is no evidence that the participants in the separate condition had any difficulty producing separate retrieval sets (as in the last experiment, there was only a single instance of a cross-list intrusion).

For a third time, we failed to demonstrate the encoding $\times$ recall interaction predicted by the retrieval account. Experiment 3 blocked the presentation of the items by category to make the categorical nature of the items more salient. Category-based clustering produced a mean ARC score of .28, which was significantly different from zero $[t(13)=4.63]$. Additional support for category-based clustering comes from 2 of 16 participants who spontaneously organized their recorded answers in two category groupings. Although it is not possible to calculate the standard ARC score for these participants, they clearly organized their recall around category information. List-based clustering was still significant [mean ARC $=.15 ; t(13)=2.63$ ] for the 14 participants for whom it was possible to compute ARC scores. Finally, the propensity to organize recall around category or lists was assessed with a sign test, comparing the magnitudes of category-based and listbased ARC scores. This was necessary because of the 2 participants for whom ARC scores could not be computed. Because these participants overtly organized recall around categories, we recorded their sign as indicating more category-based than list-based clustering. For the 16 participants in the combined condition, 12 produced more category-based than list-based clustering, 3 the reverse, and $1 \mathrm{a}$ tie, a significant difference by a sign test. This indicates that blocking by category made category structure more salient and influential during retrieval than did list structure.

\section{EXPERIMENT 4}

Although, by and large, the results from the first three experiments conform to the expectations of current encoding accounts, the main effect of encoding in Experiment 2 requires comment. Here, SPTs were better recalled than VTs. Although the enactment effect is typically more robust in within-subjects, mixed-list designs (e.g., Engelkamp \& Dehn, 2000; Engelkamp \& Zimmer, 1984; Steffens, 1999; Steffens et al., 2003), the enactment effect can also be found in between-subjects and pure-list designs (e.g., Earles \& Kersten, 2002; Koriat \& Pearlman-Avnion, 2003). The more puzzling question is why the SPT effect was significant in Experiment 2 and not in Experiments 1 and 3. In particular, with encoding accounts, there is no particular basis for expecting a larger SPT effect when the study lists are randomly ordered (Experiment 2), as opposed to blocked by category (Experiment 3 ). However, a closer examination of the data reveals that the difference in experiments may be more apparent than real. Both Experiments 1 and 3 had numerical trends for greater recall in the SPT than in the VT condition, although the differences were nonsignificant. The data from all three experiments were submitted to a $2 \times 2 \times 3$ ANOVA, using encoding condition (SPT or VT), recall condition (combined or separate), and experiment (Experiment 1, 2, or 3) as factors. Interestingly, there was a main effect of encoding such that SPT recall was greater than VT recall $[F(1,90)=7.24$, $\left.M S_{\mathrm{e}}=29.30\right]$. No other main effects or interactions were significant, including the two-way interaction between encoding condition and experiment $(F \mathrm{~s}<1)$. The results from this analysis are important for two reasons. First, the lack of a significant interaction indicates that the results of Experiment 2 were not substantially different from those of Experiments 1 and 3. Second, the overall main effect of encoding is important for establishing that our materials are capable of producing an SPT effect. Of course, the Experiment 1 materials had already demonstrated an SPT effect in Mulligan and Hornstein (2003).

Although the combined analysis indicates no differences in the SPT effect across the experiments, a crossexperimental analysis is not the ideal manner in which to resolve this issue. Experiment 4 directly tested this claim and experimentally contrasted the conditions in Experiments 2 and 3. This provided a more appropriate experimental test of whether the SPT effect truly is greater when the category items are randomly intermixed versus blocked. In addition, this experiment contributed participants for subsequent power analyses and for ancillary analyses, to be reported after Experiment 4.

\section{Method}

Participants. Sixty-four undergraduates at the University of North Carolina at Chapel Hill participated in exchange for credit in psychology courses.

Design, Materials, and Procedure. The participants were randomly assigned to one of four conditions corresponding to each of 
Table 2

Experiment 4: Mean Proportions (With Standard Deviations) of Items Recalled As a Function of Encoding and Recall Conditions

\begin{tabular}{llllll}
\hline & \multicolumn{3}{c}{ Encoding Condition } \\
\cline { 2 - 3 } \cline { 5 - 6 } Recall Condition & \multicolumn{2}{c}{ SPT } & & \multicolumn{2}{c}{ VT } \\
\cline { 2 - 3 } \cline { 5 - 6 } & $M$ & $S D$ & & $M$ & $S D$ \\
\hline Separate (blocked) & .55 & .07 & & .41 & .12 \\
Combined (blocked) & .53 & .15 & & .48 & .13 \\
Separate (mixed) & .53 & .15 & .43 & .15 \\
Combined (mixed) & .46 & .12 & .44 & .15
\end{tabular}

Note-SPT, subject-performed task; VT, verbal task.

the two conditions in the previous two experiments: (1) random list, combined recall, (2) random list, separate recall, (3) blocked list, combined recall, and (4) blocked list, separate recall. All the materials were the same as those used in Experiments 2 and 3.

\section{Results and Discussion}

The proportions recalled (Table 2 ) were submitted to a $2 \times 2 \times 2$ ANOVA, using encoding condition (SPT vs. VT), list structure (random vs. blocked), and recall (separate vs. combined) as factors. The analysis indicated a significant main effect of encoding condition, such that SPT was greater than VT $\left[F(1,63)=6.99, M S_{\mathrm{e}}=35.07\right]$, although no other main effects or interactions were significant (all $p \mathrm{~s}>.2$ ). Again, instances of cross-list intrusions were uncommon; across the 32 participants in the separate recall condition, 7 participants produced a total of eight such intrusions.

Category-based and list-based ARC scores were computed for the participants in the combined condition (Table 3). Category clustering (mean ARC $=.31$ ) was significantly greater than list-based clustering [mean $\mathrm{ARC}=$ $\left..10 ; F(1,28)=13.2, M S_{\mathrm{e}}=.59\right]$. This provides further support that our categorical manipulation of the stimuli was successful in inducing category clustering and, thus, a combined retrieval set. The interaction between ARC type (list vs. categorical) and encoding condition (blocked vs. random) was nonsignificant $(F \mathrm{~s}<1)$, indicating higher category- than list-based clustering in both the random and the blocked conditions. This contrasts somewhat with the results of Experiments 2 and 3, in which the ARC difference was clearly found only for the blocked condition (Experiment 3). Given the larger goal of inducing categorybased retrieval, however, the experiment satisfies our requirements: The use of categorized materials induced greater category clustering than list-based clustering.

The present results are important for several reasons. First and foremost, along with Experiment 2, this is a second demonstration of an SPT effect (SPT phrases recalled

Table 3

Experiment 4: Mean ARC Scores of Combined Recall Participants As a Function of Encoding Condition

\begin{tabular}{ccc}
\hline \multirow{2}{*}{$\begin{array}{c}\text { Encoding } \\
\text { Condition }\end{array}$} & \multicolumn{2}{c}{ Mean ARC Score } \\
\cline { 2 - 3 } & List & Category \\
\hline Random & .08 & .31 \\
Blocked & .13 & .30 \\
\hline
\end{tabular}

Note-ARC Score, adjusted-ratio-of-clustering score. more than VT phrases), indicating that the materials used were sensitive enough to demonstrate such an effect. Second, because the SPT effect did not differ across the mixed and blocked study lists, the notion that Experiment 2 was in any way different from the other experiments is ruled out. Instead, as the pooled analysis suggests, each of the previous experiments trended in the direction of an SPT effect, although Experiment 2 just happened to cross the significance threshold. Third, this is a fourth demonstration that the size of the SPT effect does not vary as a function of the recall condition. Finally, it might seem surprising that list organization did not enhance recall, as it often does with word lists (e.g., Hunt \& Einstein, 1981). However, this is consistent with prior research on action memory. Organization based on taxonomic categories of the action objects, as in the present experiment, does not appear to affect recall (e.g., Engelkamp, Seiler, \& Zimmer, 2004; Engelkamp \& Zimmer, 1996), although organization based on scripts (e.g., doing laundry) enhances action memory (Engelkamp \& Zimmer, 2002).

\section{ANCILLARY ANALYSES OF THE RECALL DATA}

A number of analyses are best addressed by combining data across the four experiments. First, the retrieval account predicts an interaction between encoding and test conditions, which we repeatedly failed to find. To provide a more powerful analysis, the data from all four of the recall experiments were pooled. This analysis exhibited no hint of an interaction between encoding and recall conditions $(F<1)$, despite the large sample $(N=160)$. This is a null interaction, so considering power is important. Our pooled analysis has more participants (and thus, more power) than did the original study by McDaniel et al. (2005, Experiment 1), which showed results consistent with the retrieval account. Given the size of the effect ( $f=$ .48) reported by McDaniel et al. (2005, Experiment 1), the presented analysis has power exceeding .99. Furthermore, for an effect one half that size, the power was .91. Thus, there was substantial power to find an effect even much smaller than that uncovered in McDaniel et al. (2005).

The use of ARC scores was employed to measure how much the participants clustered their responses on the basis of list (Experiments 1-4), as well as category (Experiments 2-4). The goal was to verify that the participants in the combined condition were, in fact, creating a combined retrieval set, the only way to provide an appropriate test of the retrieval hypothesis. We explored this issue further by limiting our analyses to only those participants who had list-based ARC scores of 0 or less $(n=23)$, indicating no hint of list-based clustering in the combined condition. The recall scores from these participants were compared with those from all the participants (Experiments $1-4)$ from the separate recall condition $(n=80)$, and there was no interaction between encoding and recall conditions $(F<1)$. Similarly, we examined only those participants who had category-based ARC scores above 0 ( $n=44)$ from Experiments 2-4, and still there was no 
interaction $(F=1.15)$. Perhaps the most stringent comparison, however, is an overlap of the previous two groups: those participants (Experiments 2-4) who had list-based ARC scores of 0 or lower and category-based ARC scores greater than $0(n=12)$. The ARC scores for these participants would suggest that they were the most likely to have formed a combined retrieval set, yet still there is no hint of an encoding $\times$ recall interaction $(F<1)$. The size of the SPT effect in this group is .06, whereas the size of the SPT effect in the separate group is .07, indicating no trend toward an interaction.

Researchers investigating the item order account typically use the input-output correspondence (IO) score to assess order information in free recall (DeLosh \& McDaniel, 1996; McDaniel et al., 2005; Nairne et al., 1991). This measure assesses the extent to which recall output corresponds to serial input position, with a chance-level value of .5. In the present case, this measure is likely to be suggestive rather than definitive, since IO scores tend to be near chance level for lists of 16 items or more, indicative of little reliance on serial order information (Engelkamp, Jahn, \& Seiler, 2003; Mulligan \& Lozito, 2007). In the combined condition, the two study lists were treated as a single list for purposes of computing IO. In the separate condition, an IO score was computed for each test, along with an average of the two to facilitate comparison with the combined condition. To compute an IO score, at least two items must be recalled, preventing computation for some of the recall protocols in the separate condition. In general, the results of the IO scores were as expected. First, average IO scores were very low but significantly greater than chance [with an overall average of .52; $t(150)=$ 2.72]. Second, IO scores were significantly above chance in the separate condition [averaged over tests; $M=.54$; $t(79)=2.72]$ but not significantly different from chance in the combined condition $[M=.50 ; t(69)=0.29]$. Given that the functional study list was longer in the combined condition, this pattern is consistent with the finding that longer lists exhibit less IO correspondence (Mulligan \& Lozito, 2007). Third, for the separate condition, IO scores for the VT condition exceeded chance $[M=.56 ; t(79)=$ 3.04], whereas those for the SPT condition did not $[M=$ $.52 ; t(79)=1.63]$. This is consistent with the item order account, which argues for deficient order memory in the SPT condition but is merely suggestive, since the direct comparison between these conditions was not significant $[t(79)=1.56, p=.12]$.

\section{GENERAL DISCUSSION}

A number of memory effects (e.g., bizarreness, enactment, generation, perceptual identification, word frequency) are modulated by experimental design. These effects share other similarities and have been analyzed with common theories, some focusing on encoding processes (e.g., Engelkamp \& Dehn, 2000; McDaniel \& Bugg, 2008; Mulligan \& Lozito, 2004; Nairne et al., 1991; Steffens, 2007) and others focusing on retrieval (Hunt \& Worthen, 2006; McDaniel et al., 2005; see Schmidt, 2007, for a discussion). Both types of theories provide accounts of design effects, but the usual experimental paradigm for studying design effects confounds encoding and retrieval context. McDaniel et al. (2005) addressed this problem with a new paradigm and found support for a retrieval account of the bizarreness effect.

The present experiments investigated whether this retrieval-based analysis applies to the enactment effect. This account proposes that retrieval contributes to the enactment effect and that the effect should be enhanced by a combined, rather than pure, retrieval set. Encoding accounts (e.g., the item-specific-relational account, Engelkamp's item-specific motoric account) generally imply that recall condition should not influence the enactment effect, because the encoding conditions were held constant. Using the paradigm developed by McDaniel et al. (2005), we failed to support the retrieval account. Consistently across four experiments, instructing participants to recall both lists in a combined recall did not yield a larger enactment effect than did having them perform two separate recalls.

It should be noted that throughout the four experiments, efforts were made to adequately assess the retrieval account. First, we made use of the paradigm developed by McDaniel et al. (2005), which was designed to assess the retrieval account and is capable of producing results supportive of that account. Second, when the initial experiment yielded results contrary to the retrieval account, we examined additional assumptions that might render the results consistent with the retrieval account. Specifically, we examined whether the lists were not discriminable enough to allow separate retrieval sets in the separate test condition. The very low level of cross-list intrusions indicates that this was not a problem. Next, we entertained the idea that the study lists were too easily discriminated, which might undermine the combined retrieval instructions. Experiments 2-4 used categorized materials spread across the study lists to ensure a cross-list, combined retrieval set. After having given the retrieval account these additional benefits of the doubt, the results were still inconsistent with the retrieval account. Even when pooling together data from all four experiments in a high-powered analysis, there is still no hint of the predicted interaction between recall and encoding condition. The average size of the enactment effect was similar across the retrieval conditions and, in fact, was (nonsignificantly) greater in the separate condition. Thus, the experiments provide no evidence that retrieval processes drive design effects in the case of enactment.

The present experiments were designed to assess the retrieval account, rather than test differences among various encoding accounts. From the perspective of the encoding accounts, however, the results are generally as expected: Because nothing varied at encoding, the enactment effect was expected to be comparable across recall groups. However, it should be noted that although these accounts focus primarily on encoding differences between the SPT and VT conditions, the item order account also describes how retrieval conditions can contribute to the appearance 
of the effect (i.e., this account is not a "pure" encoding account). This may complicate the interpretation of results with regard to this particular encoding account in one instance. Specifically, the item order account argues that order information is an important determinant of recall under some conditions (e.g., shorter lists, unrelated study materials) but not others (e.g., longer lists, categorized materials) (e.g., McDaniel \& Bugg, 2008; Mulligan \& Lozito, 2007; Nairne et al., 1991). Consequently, this account proposes that the order-encoding deficit for the unusual condition (e.g., generation, SPT) impairs recall of these items predominantly when order-based retrieval strategies are used. In the present experimental paradigm, the item order account implies that when short lists of unrelated study materials are used, the SPT effect may be smaller in the separate than in the combined test condition, because the former condition requires recall of a functionally shorter list, making order-based recall more likely. Under these conditions, the item order account appears to make the same prediction as the retrieval account: a larger SPT effect in the combined than in the separate condition. When longer lists or related (e.g., categorized) study materials are used, the item order account does not predict this interaction. Under these conditions, the item order account makes the generic encoding prediction that changes from the separate to the combined retrieval condition should not moderate the SPT effect. It should be noted that the IO scores were not much above chance, and key comparisons were not significantly different, giving little indication that order information was a key determinant of recall (not unexpected, given the use of action phrases and the length of the study lists used; Engelkamp et al., 2003; Mulligan \& Lozito, 2007). Finally, the other encoding accounts (the item-specific-relational account, Engelkamp's item-specific motoric view) do not specify retrieval conditions that modify their predictions, so the present results may be taken as consistent with these theories in a more straightforward way.

The present results raise the question as to why the results of McDaniel et al. (2005), who originally investigated bizarreness, do not extend to other, similar memory phenomena. Mulligan and Peterson (2008) examined the generation and perceptual interference effects with an experimental paradigm similar to that in the present study and found similar results; combined retrieval conditions did not enhance the size of either effect. That is, the predictions of the retrieval hypothesis were not observed for either generation or perceptual interference. These four effects (bizarreness, enactment, generation, and perceptual interference) behave similarly to one another in response to many experimental manipulations, which has motivated common explanations for all of them (e.g., McDaniel \& Bugg, 2008). The fact that the retrieval account, which fits the data from McDaniel et al. (2005) so well, does not extend to other, similar phenomena is concerning and complicates attempts to model all of these phenomena as having a common underlying explanation.

Mulligan and Peterson (2008) speculated that differences in the nature of the study items between their experiments and McDaniel et al.'s (2005) experiments might contribute to the divergent results. In Mulligan and Peterson's experiments, participants were presented with words in isolation, whereas in McDaniel et al.'s (2005) experiments, participants saw complete sentences. Mulligan and Peterson speculated that perhaps more complex items produce a greater reliance on retrieval processes for bizarreness. In addition, Mulligan and Peterson pointed to evidence that it is easier to form interitem associations with random lists of simple items, which might render encoding factors more determinative with such materials. Critically, in the present experiments, neither of these explanations seems likely, since the action phrases were much like the bizarre sentences (in terms of structure and complexity) used in McDaniel et al. (2005). As such, differences in the nature of the study items do not seem to drive the differences found between generation, perceptual interference, and enactment, on the one hand, and bizarreness, on the other. McDaniel and Bugg (2008) speculated that bizarre sentences may produce a greater reliance on item distinctiveness in recall, consistent with findings that the bizarreness effect is more malleable in the face of retrieval manipulations than are the other manipulations (enactment, generation, and perceptual interference; see Mulligan \& Peterson, 2008, p. 1380, for a discussion). Clearly, additional research is required to determine why this is the case.

\section{CONCLUSIONS}

A number of memory phenomena are modulated by experimental design, with a greater effect (e.g., of bizarreness, enactment, generation, perceptual interference) in recall for mixed-list than for pure-list designs. Traditionally, encoding explanations have been offered to explain these effects, although recent evidence suggests that retrieval may also play a role (McDaniel et al., 2005). The present study applied a newly created experimental paradigm, developed by McDaniel et al. (2005), to specifically test whether or not factors operating at retrieval could explain the enactment effect. Across a series of four experiments, manipulating the manner in which participants recalled a series of action phrases had no impact on the size of the enactment effect. As such, these results do not provide support for the retrieval account.

\section{AUTHOR NOTE}

Correspondence concerning this article should be addressed to N. W. Mulligan, Department of Psychology, University of North Carolina, Chapel Hill, NC 27599-3270 (e-mail: nmulligan@unc.edu).

\section{REFERENCES}

BäcKman, L., \& NiLsson, L.-G. (1984). Aging effects in free recall: An exception to the rule. Human Learning, 3, 53-69.

Bä́ckman, L., Nilsson, L.-G., \& Chalom, D. (1986). New evidence on the nature of the encoding of action events. Memory \& Cognition, 14, 339-346.

BegG, I., Snider, A., Foley, F., \& Goddard, R. (1989). The generation effect is no artifact: Generating makes words distinctive. Journal of Experimental Psychology: Learning, Memory, \& Cognition, 15, 977-989. 
CoHEN, R. L. (1981). On the generality of some memory laws. Scandinavian Journal of Psychology, 22, 267-281.

CoHen, R. L. (1989). Memory for action events: The power of enactment. Educational Psychology Review, 1, 57-80.

DeLosh, E. L., \& McDaniel, M. A. (1996). The role of order information in free recall: Application to the word-frequency effect. Journal of Experimental Psychology: Learning, Memory, \& Cognition, 22, 1136-1146.

Earles, J. L., \& Kersten, A. W. (2002). Directed forgetting of actions by younger and older adults. Psychonomic Bulletin \& Review, 9, 383388.

EngelKamp, J. (1998). Memory for actions. Hove, U.K.: Psychology Press/Taylor \& Francis.

EngelKamp, J., \& Dehn, D. M. (2000). Item and order information in subject-performed tasks and experimenter-performed tasks. Journal of Experimental Psychology: Learning, Memory, \& Cognition, 26, 671-682.

Engelkamp, J., Jahn, P., \& Seiler, K. H. (2003). The item-order hypothesis reconsidered: The role of order information in free recall. Psychological Research, 67, 280-290.

EngelKamp, J., Seiler, K. H., \& Zimmer, H. D. (2004). Memory for actions: Item and relational information in categorized lists. Psychological Research, 69, 1-10.

ENGELKAMP, J., \& ZiMMER, H. D. (1984). Motor programme information as a separable memory unit. Psychological Research, 46, 283-299.

EngelKamp, J., \& Zimmer, H. D. (1985). Motor programs and their relation to semantic memory. German Journal of Psychology, 9, 239254.

ENGelKamp, J., \& Zimmer, H. [D.] (1996). Organisation and recall in verbal tasks and in subject-performed tasks. European Journal of Cognitive Psychology, 8, 257-273.

ENGELKAMP, J., \& ZiMMER, H. D. (2002). Free recall and organization as a function of varying relational encoding in action memory. Psychological Research, 66, 91-98.

Engelkamp, J., Zimmer, H. D., Mohr, G., \& Sellen, O. (1994). Memory of self-performed tasks: Self-performing during recognition. Memory \& Cognition, 22, 34-39.

Hunt, R. R., \& EINSTEIN, G. O. (1981). Relational and item-specific information in memory. Journal of Verbal Learning \& Verbal Behavior, 19, 497-514.

Hunt, R. R., \& McDaniel, M. A. (1993). The enigma of organization and distinctiveness. Journal of Memory \& Language, 32, 421-445.

Hunt, R. R., \& Worthen, J. B. (2006). Distinctiveness and memory. New York: Oxford University Press.

Koriat, A., \& Pearlman-Avnion, S. (2003). Memory organization of action events and its relationship to memory performance. Journal of Experimental Psychology: General, 132, 435-454.

KormI-NourI, R. (1995). The nature of memory for action events: An episodic integration view. European Journal of Cognitive Psychology, 7, 337-363.

Kormi-NOurI, R. (2000). The role of movement and object in action memory: A comparative study between blind, blindfolded and sighted subjects. Scandinavian Journal of Psychology, 41, 71-75.

MCDANIEL, M. A., \& BUGG, J. M. (2008). Instability in memory phenomena: A common puzzle and a unifying explanation. Psychonomic Bulletin \& Review, 15, 237-255.

McDaniel, M. A., DeLosh, E. L., \& Merritt, P. S. (2000). Order information and retrieval distinctiveness: Recall of common versus bizarre material. Journal of Experimental Psychology: Learning, Memory, \& Cognition, 26, 1045-1056.
McDaniel, M. A., Dornburg, C. C., \& Guynn, M. J. (2005). Disentangling encoding versus retrieval explanations of the bizarreness effect: Implications for distinctiveness. Memory \& Cognition, 33, 270-279.

Mulligan, N. W. (1999). The effects of perceptual interference at encoding on organization and order: Investigating the roles of itemspecific and relational information. Journal of Experimental Psychology: Learning, Memory, \& Cognition, 25, 54-69.

Mulligan, N. W. (2006). Hypermnesia and total retrieval time. Memory, 14, 502-518.

Mulligan, N. W., \& Hornstein, S. L. (2003). Memory for actions: Self-performed tasks and the reenactment effect. Memory \& Cognition, 31, 412-421.

Mulligan, N. W., \& Lozito, J. P. (2004). Self-generation and memory. In B. H. Ross (Ed.), The psychology of learning and motivation: Advances in research and theory (Vol. 45, pp. 175-214). San Diego: Elsevier.

Mulligan, N. W., \& Lozito, J. P. (2007). Order information and free recall: Evaluating the item-order hypothesis. Quarterly Journal of Experimental Psychology, 60, 732-751.

Mulligan, N. W., \& Peterson, D. (2008). Assessing a retrieval account of the generation and perceptual-interference effects. Memory \& Cognition, 36, 1371-1382.

Nairne, J. S., Riegler, G. L., \& Serra, M. (1991). Dissociative effects of generation on item and order retention. Journal of Experimental Psychology: Learning, Memory, \& Cognition, 17, 702-709.

RoEDIGER, H. L., III (2008). Relativity of remembering: Why the laws of memory vanished. Annual Review of Psychology, 59, 225-254.

Roenker, D. L., Thompson, C. P., \& Brown, S. C. (1971). Comparison of measures for the estimation of clustering in free recall. Psychological Bulletin, 76, 45-48.

SCHMIDT, S. R. (2007). Unscrambling the effects of emotion and distinctiveness on memory. In J. S. Nairne (Ed.), The foundations of remembering: Essays in honor of Henry L. Roediger III (pp. 141-158). New York: Psychology Press.

STEFFENS, M. C. (1999). The role of relational processing in memory for actions: A negative enactment effect in free recall. Quarterly Journal of Experimental Psychology, 52A, 877-903.

StefFens, M. C. (2007). Memory for goal-directed sequences of actions: Is doing better than seeing? Psychonomic Bulletin \& Review, 14, 1194-1198.

Steffens, M. C., Buchner, A., \& Wender, K. F. (2003). Quite ordinary retrieval cues may determine free recall of actions. Journal of Memory \& Language, 48, 399-415.

Steffens, M. C., Buchner, A., Wender, K. F., \& Decker, C. (2007). Limits on the role of retrieval cues in memory for actions: Enactment effects in the absence of object cues in the environment. Memory \& Cognition, 35, 1841-1853.

Tulving, E., \& Thomson, D. M. (1973). Encoding specificity and retrieval processes in episodic memory. Psychological Review, 80, 352-373.

\section{NOTES}

1. All items involved real objects that were subsequently removed after each item presentation, leaving no retrieval cues available at test (Steffens et al., 2003; Steffens, Buchner, Wender, \& Decker, 2007).

2. Preliminary analyses of the separate condition indicated that recall on the first and second tests did not significantly differ, and test order (SPT list tested first vs. VT list tested first) did not produce a significant main effect or interaction. This pattern was obtained for this and all the subsequent experiments $\left(F_{\mathrm{S}}<1.2\right)$. 
APPENDIX

\section{Actions Used in Experiment 1}

ring the bell

put Easter egg together

fold the shirt

close the purse

drop the pencil

push the toy car

place tube standing up

click the computer mouse

shuffle the cards

rattle the keys

dial a telephone number

crumble the plastic bag

undo safety pin

stretch the elastic band

turn over the coin

pick up the battery

tear the paper

remove tape from case

roll the marble

bend the paper clip

shoot the toy gun

tie a knot using the string

spin the top

break the match

squeeze the dog toy

wave a handkerchief

bounce the ball

pat the dog

open the book

press the stapler

unzip the zipper

seal the envelope

take cap off pen

unfold the napkin

stack the checkers

make twist tie into a $\mathrm{V}$

smell the flower

wring out a sponge

shake the bottle

turn on the flashlight

\section{Actions Used in Experiments 2 and 3}

twist the elastic band

drop the pencil

peel off label

grasp the marker

slide the binder clip

take the cap off the pen

fold the index card

turn over the clipboard

bend the paper clip

tear the paper

undo the puzzle pieces

shuffle the cards

toss the jack in the air

shake the Magic 8-Ball

wind the yo-yo

wave the pinwheel

bounce the ball

flip the Frisbee

push the toy car

lift the doll pat the dog

jump the checkers

press the stapler

squeeze the hole punch

tap the ruler against the table

open the book

pull a Post-it note from its stack

remove a piece of tape from its case

click the computer mouse

close the notebook

seal the envelope

pick up the eraser

shoot the toy gun

stretch the Slinky

stack the blocks

rub the rubber ducky

put together the Legos

place the action figure standing up roll the marble spin the top

(Manuscript received April 17, 2009;

revision accepted for publication August 28, 2009.) 